Supplement of Atmos. Chem. Phys., 20, 1301-1316, 2020

https://doi.org/10.5194/acp-20-1301-2020-supplement

(C) Author(s) 2020. This work is distributed under

the Creative Commons Attribution 4.0 License.

(c) (1)

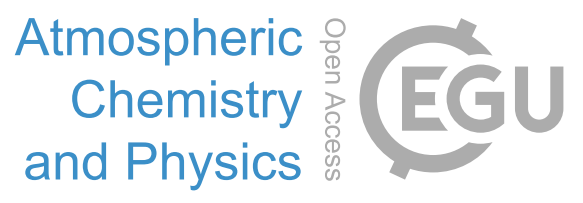

Supplement of

\title{
The impact of secondary ice production on Arctic stratocumulus
}

\section{Georgia Sotiropoulou et al.}

Correspondence to: Georgia Sotiropoulou (georgia.sotiropoulou@epfl.ch) and Athanasios Nenes (athanasios.nenes@epfl.ch)

The copyright of individual parts of the supplement might differ from the CC BY 4.0 License. 


\section{Contents of this file}

Following a brief introduction, this document includes additional technical information on the Lagrangian Parcel Model (LPM) set-up and the ACCACIA observations used to constrain this model (Text S1, Figs S1-4). A detailed view on the LPM results (look-up tables) used to parameterize SIP in the Large Eddy Simulation (LES) is also offered in Text S2 and Figs. S5-8.

\section{Introduction}

In the main text, a LES and a LPM are combined to study the influence of three SIP mechanisms: rimesplintering (RS), collisional break-up (BR) and drop-shattering (DS) on Arctic stratocumulus clouds. The main result is that RS and BR have a very weak impact when activated alone, but when combined they can explain the observed ICNCs. DS remains completely ineffective. The text in this Supporting Information provides additional information necessary to the credibility of the LPM set-up used in the main text.

In Text S1 and Fig. S1 details on how the LPM ice nucleation rate is constrained based on the LES results are offered. Figures S2-4 offer supporting information on how the LPM initial conditions and timescales are parameterized (see main text for a discussion). Finally, Text S2 and Figures S5-8 offer a detailed view of the LPM results (look-up tables) which are used to parameterize SIP in the LES.

\section{Text S1:}

In Fig. S1 the mean profiles produced by the LES after spin-up period (no SIP is activated) are shown: the nucleation scheme (DeMott×5) predicts concentrations $\left(N_{i c e}\right)$ varying from $0.007 \mathrm{~L}^{-1}$ at cloud base $(\sim$ $-3^{\circ} \mathrm{C}$ ) to $0.11 \mathrm{~L}^{-1}$ near cloud top. An interesting finding is that $N_{\text {ice }}$ profile does not match the distribution of INPs. This is likely due to the effect of cloud mixing on ice crystals, as this more homogeneous profile develops within the first hour of simulation (not shown). If SIP was directly implemented in the LES, $N_{\text {ice }}$ profiles would be used to calculate ice-ice collisions. Hence we use the $N_{\text {ice }}$ profiles as input to the LPM: a mean INP concentration of $0.007 \mathrm{~L}^{-1}$ is prescribed at cloud base, while an INP nucleation rate is applied throughout the simulation, calculated as the product of updraft velocity, an assumed lapse rate of $6 \mathrm{~K} \mathrm{~km}^{-1}$, and the temperature derivative of the INP concentration estimated with the same nucleation scheme as in the LES. Nucleation is inhibited when a maximum value of $1.1 \mathrm{~L}^{-1}$ is reached; this is the maximum $N_{\text {ice }}$ (INP) concentration produced by the LES near cloud top (Fig. S1).

\section{Text S2:}

The LPM is run over a certain range of temperature and vertical velocities, representative of the ACCACIA conditions (Fig. S4): $T_{c b h}$ is varied between -5 and $0^{\circ} \mathrm{C}$, and $W$ between 0.25 and $1.25 \mathrm{~m} \mathrm{~s}^{-1}$, with a step value of $0.5^{\circ} \mathrm{C}$ and $0.25 \mathrm{~m} \mathrm{~s}^{-1}$, respectively. These results are used to derive the ice 
enhancement factors, which are used as look-up tables in the LES to parameterize SIP.

Figure S5 show enhancement factors from the LPM simulations which account for all SIP mechanisms. Panel (a) corresponds to simulations that employ Phillips parameterization for break-up (CNTRL), while in (b-d) BR is based on Takahashi's results scaled with a factor of (b) 10, (c) 50 and (d) 100. SIP is more efficient in low updraft conditions, $<\sim 0.5 \mathrm{~m} \mathrm{~s}^{-1}$, since decreasing $W$ elongates the lifetime of the parcel within the cloud layer, allowing for the ice crystals to rime sufficiently. Nevertheless, such weak updrafts are dominant in the LES simulations (Fig. S4). Phillips' description (Fig. S5a) produces similar results with Takahashi's formula scaled with a factor of 100, which accounts for collisions of $100-\mu \mathrm{m}$ ice particles (Fig. S5d): the maximum enhancement in these simulations is a factor 20. When scaled for even larger ice particles, Takahashi's parameterization produces enhancement factors up to 50 (Fig. S5c), or even up to 100 (Fig. S5b) when mm-particles are assumed.

Figure S6 show enhancement factors from the LPM simulations which account for only one SIP mechanism. DS remains completely inactive over the entire parameter space (Fig S6a). RS is weak, producing a maximum enhancement factor of 8 (Fig S6b). The BR parameterization in panels c, e and $\mathrm{f}$ results in even weaker multiplication than RS, which occurs only for very limited thermodynamic conditions: $W<\sim 0.5 \mathrm{~m} \mathrm{~s}^{-1}$ and $T_{c b h}<-1^{\circ} \mathrm{C}$. Break-up of mm-size ice paricles (Fig. S6c) is the only process producing substantial multiplication; the enhancement can be up to two orders of magnitude for the $T_{c b h}$ and $W$ range of interest (Fig. S4).

Figure S7 and S8 show ice enhancement factors for different CCN conditions. Increasing CCN results in more effective RS (Fig. S7a, d), but has no impact on BR efficiency (Fig. S7b, e). In low CCN conditions SIP enhancement is never larger than a factor of 10 RS (Fig. S7c), while in high CCN conditions it can be as large as a factor of 50 (Fig. S7d). Increasing INPs have a very pronounced positive impact on BR (Fig. S8b, e), while it only slightly affects RS (Fig. S8a, d). DM parameterization produces very few primary ice crystals that are not enough to initiate ice-ice collisions (Fig. S8b). $\mathrm{DM} \times 10$ enhances BR only for very limited thermodynamic conditions (Fig. S8d), but when combined with RS its efficiency in low updraft conditions and cold cloud-base temperatures is increased (Fig. S8e). This is due to the fact that the new fragments ejected due to RS can grow to sufficiently large particles to initiate more ice-ice collisions. Finally, DM×100 corresponds to rather extremely high INP conditions; the abundant primary ice crystals collide with each other frequently, resulting in a large ICNC enhancement, up to a factor of 100 (Fig. S8h, i).

\section{Figures:}




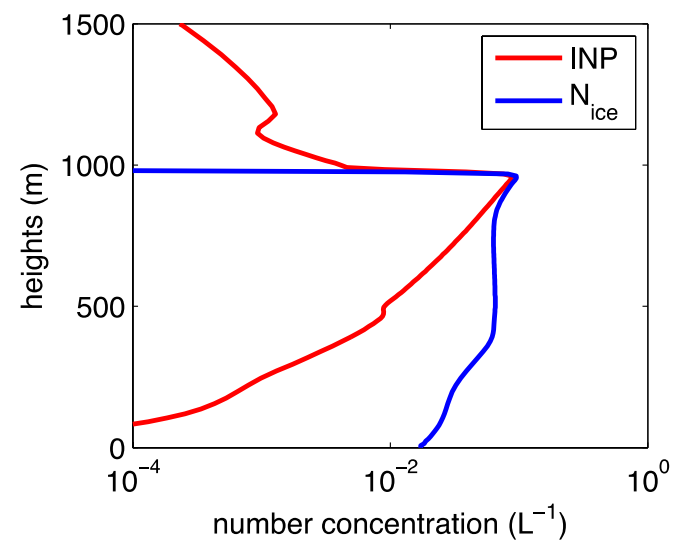

Figure S1: Mean LES profiles of INP and $N_{\text {ice }}$ concentrations from NOSIP simulation after spin-up period.
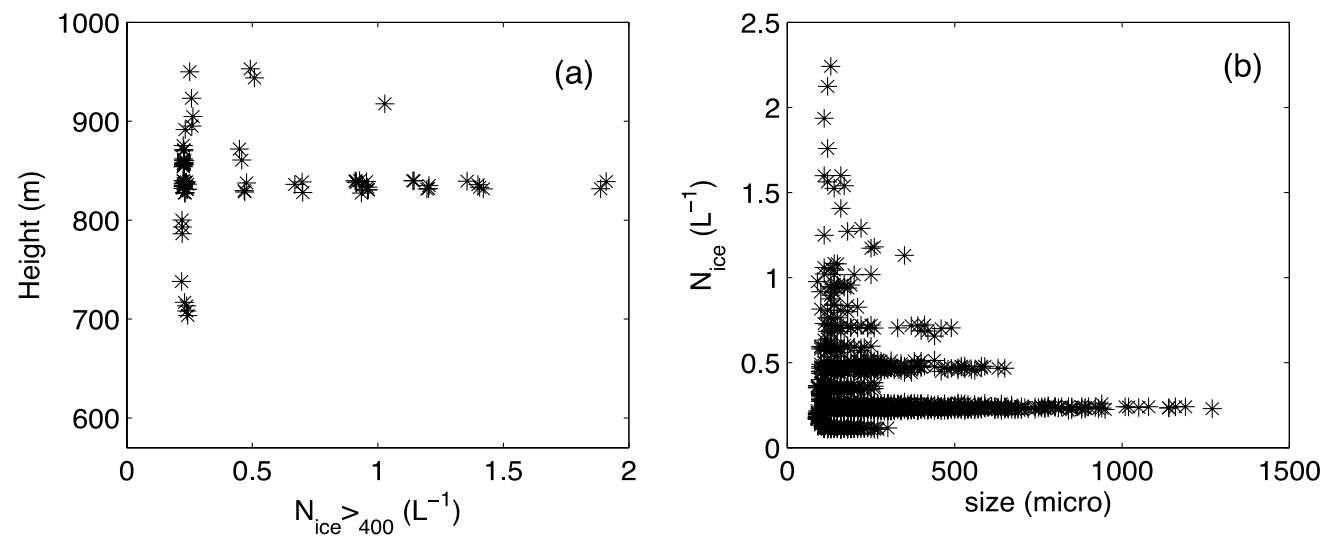

Figure S2: (a) Vertical profile of in-cloud measurements of ICNCs with diameter $\sim 400 \mu \mathrm{m}$ or larger, and (b) all ICNC measurements as a function of size. 

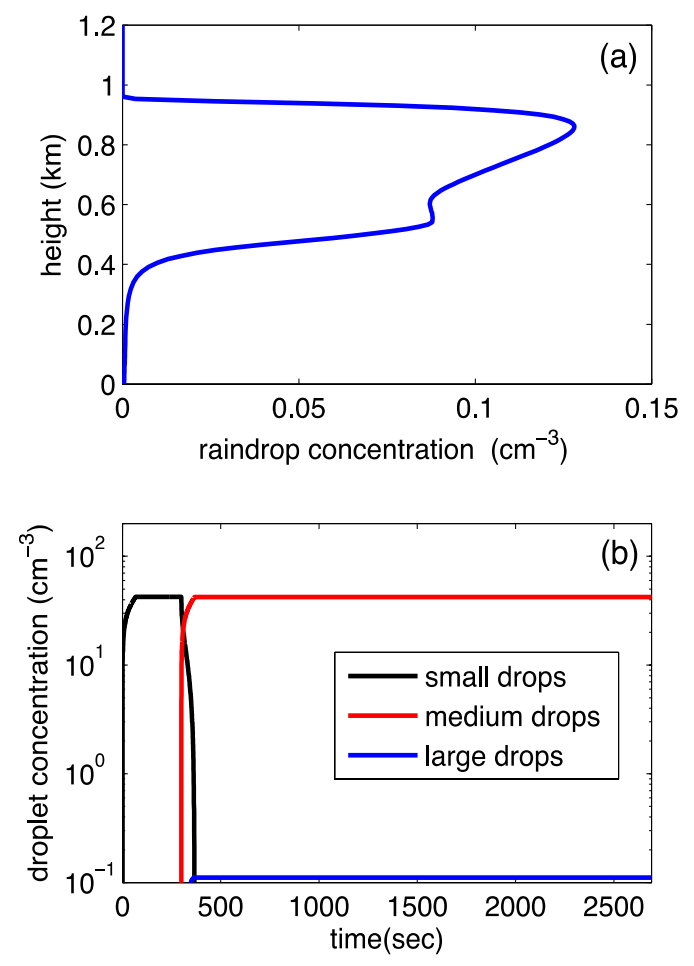

Figure S3: (a) Vertical profile of rain droplet concentration after 2.5 hours of simulation time (when maximum precipitation is observed) for the LES CNTRL simulation. (b) Timeseries of droplet concentrations for the three bins of the LPM. This LPM simulation is initialized with $T_{c b h}=-3.5^{\circ} \mathrm{C}$ and $W=0.25 \mathrm{~m} \mathrm{~s}^{-1}$ (the approximate LES mean conditions - see Fig. S4).
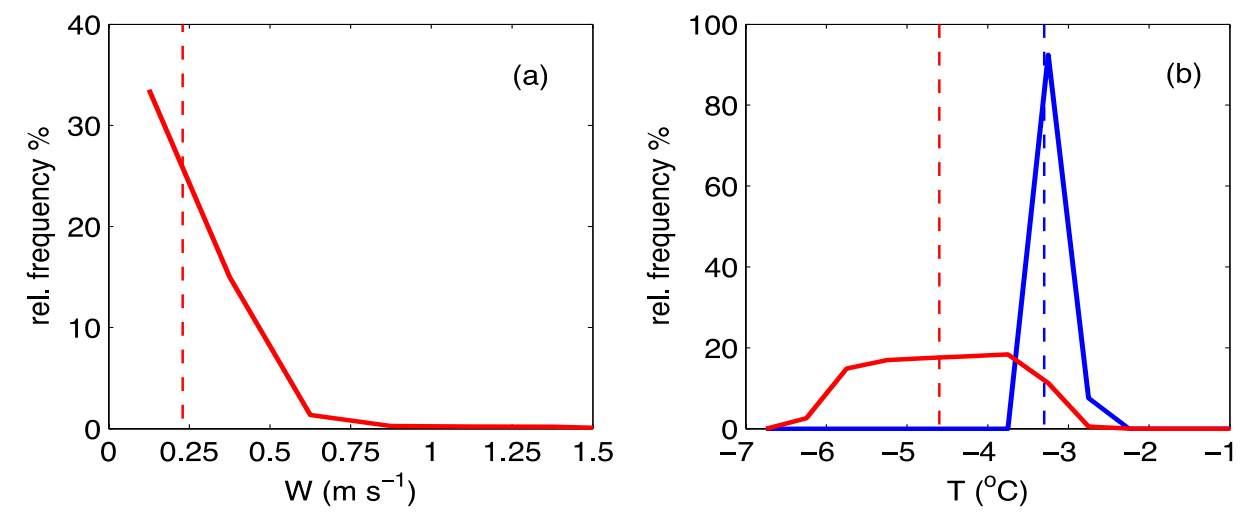

Figure S4: Relative frequency distribution of the (a) in-cloud updraft velocities $\left(W>0.05 \mathrm{~m} \mathrm{~s}^{-1}\right)$ and (b) in-cloud (red) and cloud-base (blue) temperatures derived from hourly outputs of the LES 3D fields after spin-up period. Dashed lines represent mean values. 

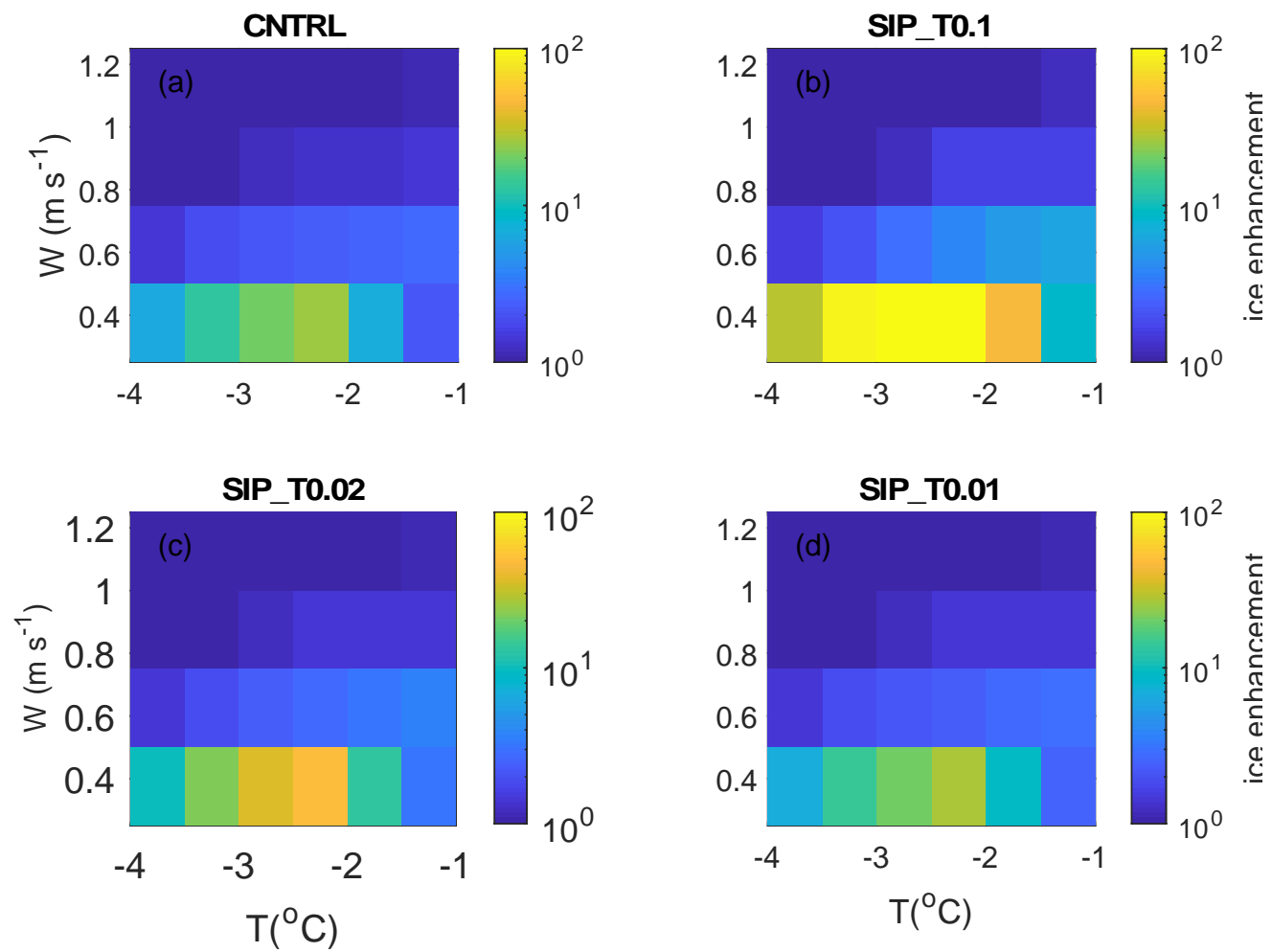

Figure S5: Enhancement factors for ice crystal number concentrations $\left(N_{i}\right)$ as a function of updraft velocity $(W)$ and cloud base temperature $\left(T_{c b h}\right)$ derived from LPM simulations with all SIP mechanisms activated. The different panels correspond to simulations that employ a BR parameterization based on (a) Phillips et al. (2017a), and on Takahashi's dataset scaled with a factor of (b) 10, (c) 50 and (d) 100. These results are used as look-up tables in the LES to parameterize secondary ice production. Note the logarithmic scale of the colorbars. 

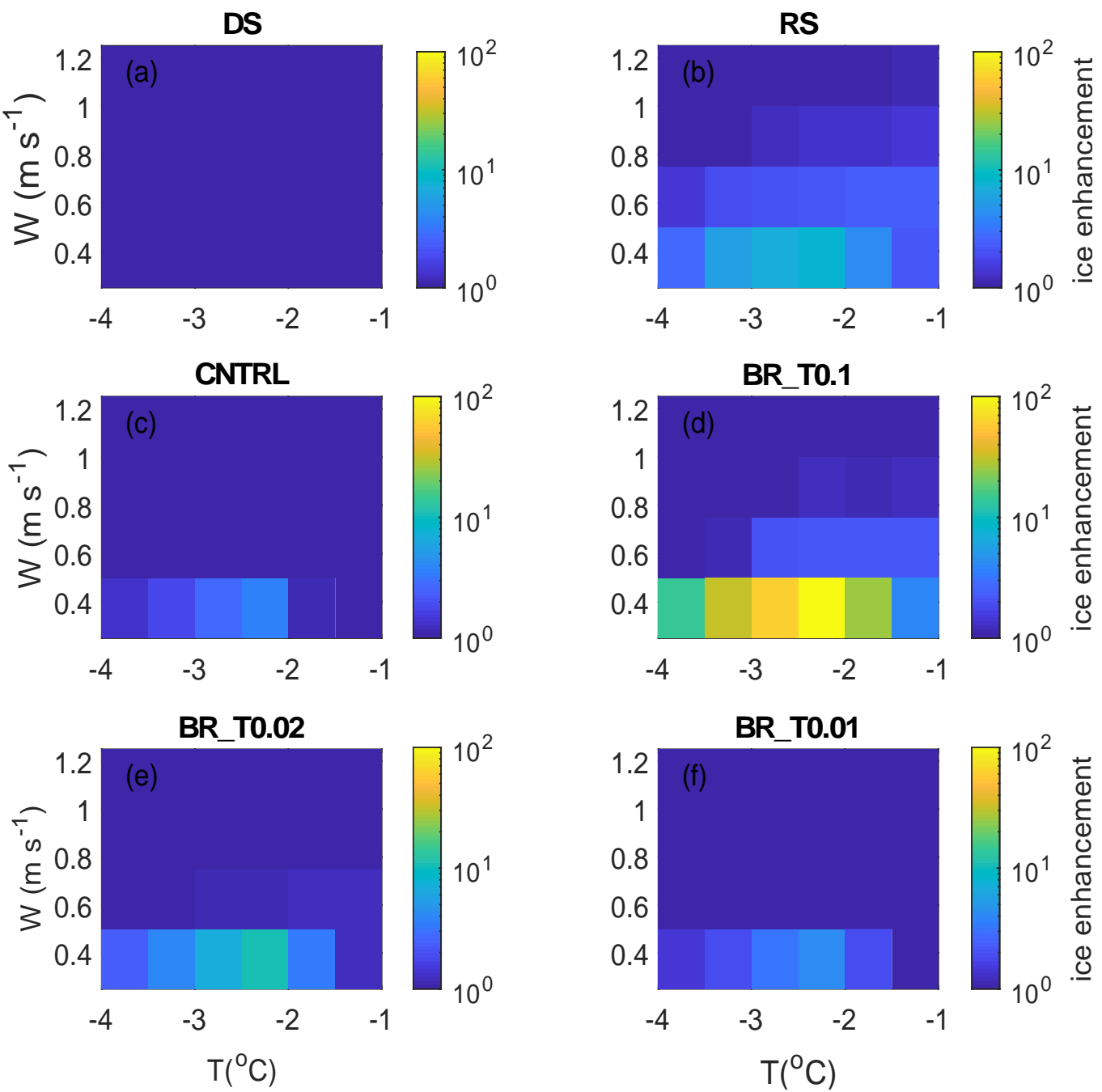

Figure S6: Same as Fig. S4 but for LPM simulations with only one active SIP mechanism. The different panels correspond to simulations in which (a) drop-shattering, (b) rime-splintering and (c-f) collisional break-up is activated. BR in (c) is parameterized after Phillips et al. (2017a), while in (d-f) the Takahashi's parameterization is used, scaled with a factor of (d)10, (e) 50 and (f) 100. 

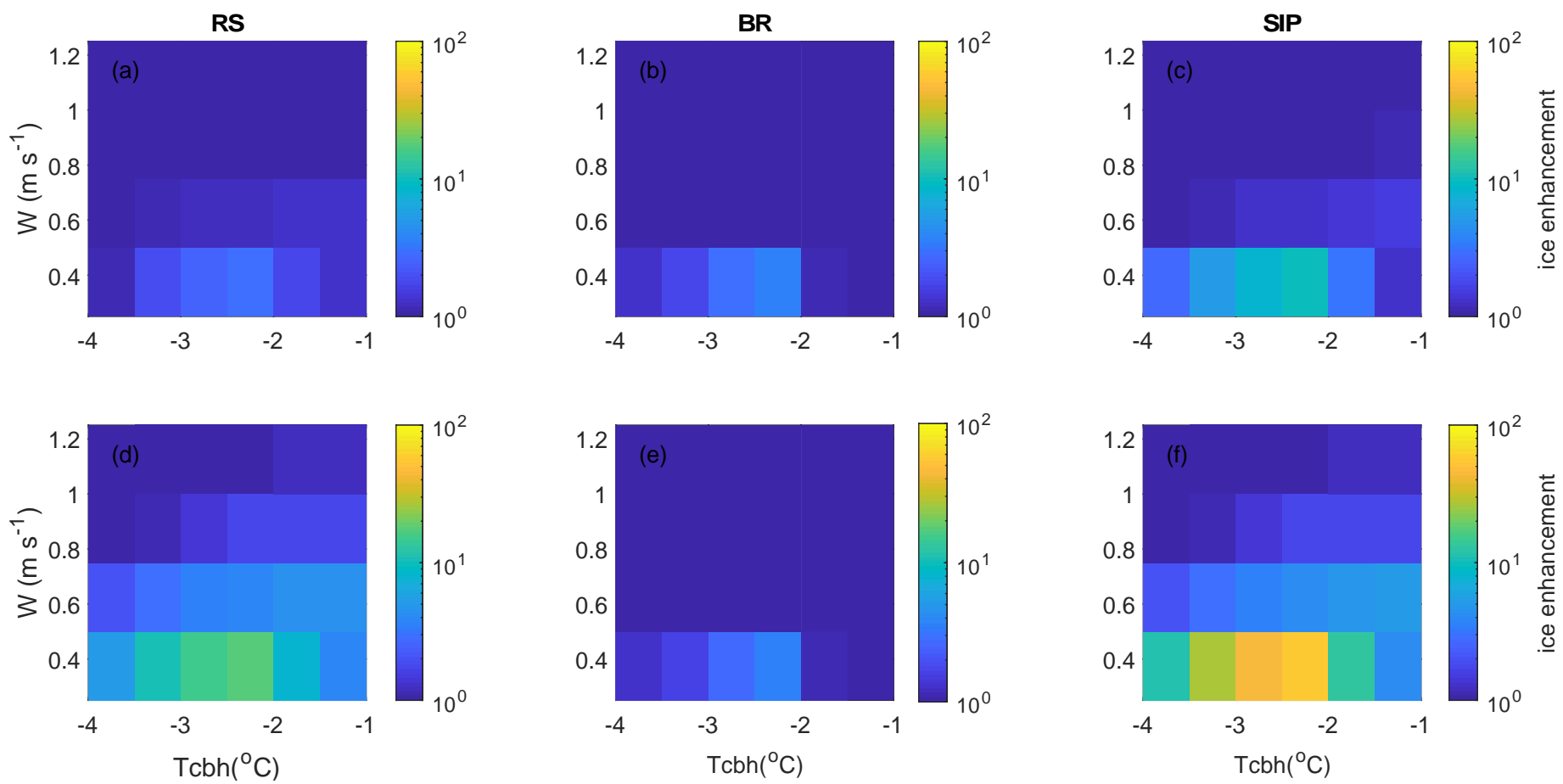

Figure S7: Same as Fig. S4 but for the LPM simulations with varying $\mathrm{N}_{\mathrm{CCN}}$ concentrations: (a-c) 10 $\mathrm{cm}^{-3}$ and (d-f) $100 \mathrm{~cm}^{-3}$. The three columns correspond to three different set-ups: (a, d) rime-splintering, (b, e) collisional break-up (Phillips et al., 2017a), and (c, f) all SIP mechanisms are activated. 

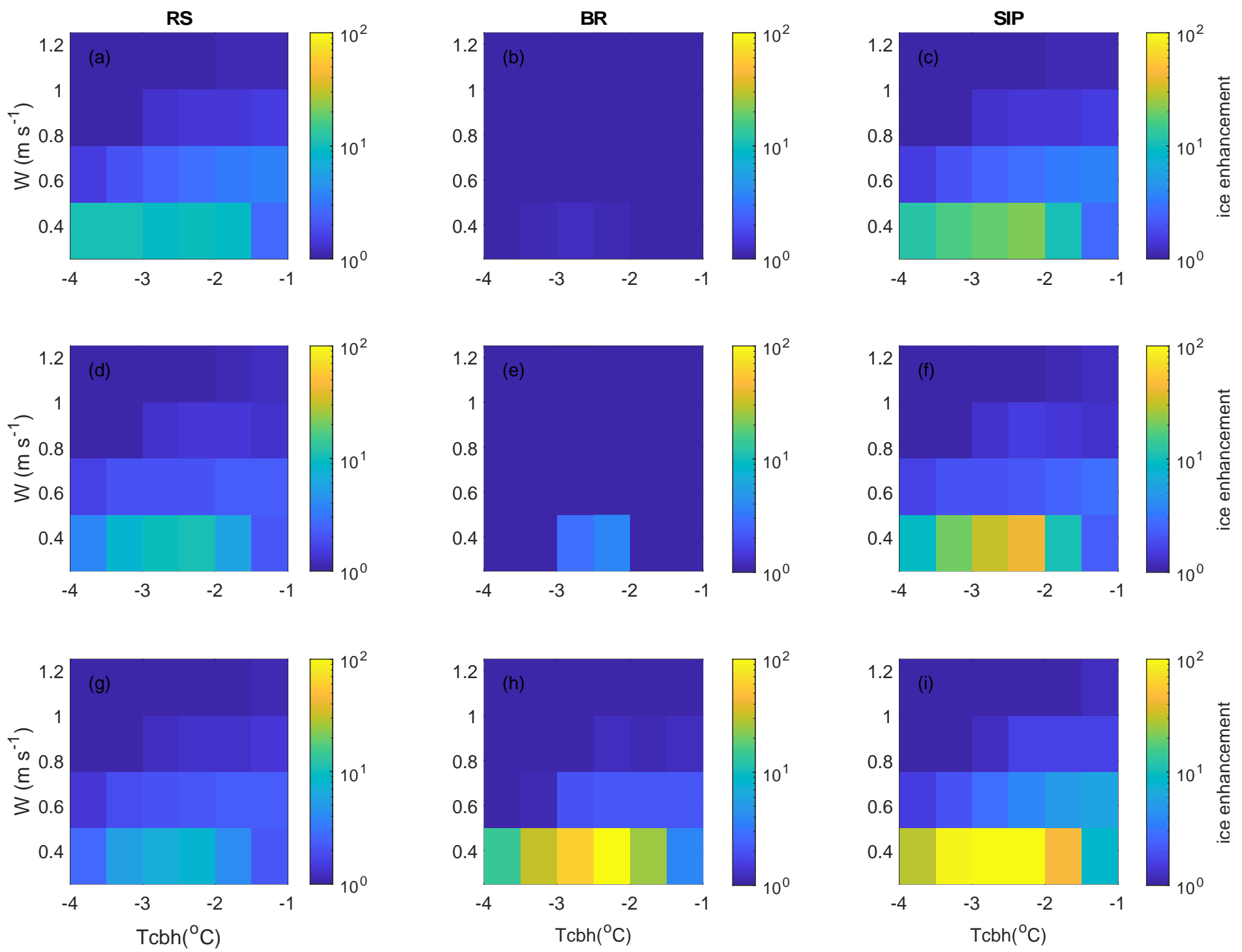

Figure S8: Same as Fig. S4 but for the LPM simulations with varying INP parameterizations: (a-c) DM and (d-f) $\mathrm{DM} \times 10$ and (g-i) $\mathrm{DM} \times 100$. The three columns correspond to three different set-ups: $(\mathrm{a}, \mathrm{d}, \mathrm{g})$ rime-splintering, (b, e, h) collisional break-up (Phillips et al., 2017a) and (c, f, i) all SIP mechanisms are activated. 